\title{
Geometric Aspects of Aleksandrov Reflection and Gradient Estimates for Parabolic Equations
}

\author{
BennetT Chow
}

\section{Introduction.}

In the first part of this paper we obtain a new gradient estimate for starshaped hypersurfaces moving in the direction of their normals with speed a function of the principal curvatures. For convex hypersurfaces moving by their curvature normal vectors, a uniform gradient estimate for the support function was obtained by Chow-Gulliver [CG1]. The proof was based on a fixed plane version of the Aleksandrov reflection method. Furthermore, in Chow-Gulliver [CG2], the Aleksandrov reflection method was applied to embedded hypersurfaces moving by their curvature vectors. In section 2 , we apply their reflection result to obtain a uniform gradient estimate for the radial function of a starshaped hypersurface moving by its curvature vector, which holds outside a certain compact set depending only on the initial hypersurface. This gradient estimate should be useful in proving long-time existence theorems for starshaped hypersurfaces expanding by nonhomogeneous functions of curvature. For homogeneous of degree one functions, long-time existence theorems were proved by Gerhardt [G] and Urbas [U2]. We also give a new geometric proof of the gradient estimate for the support function for evolving convex hypersurfaces in [CG1]. The gradient estimate for the support function was used as a first step in proving long-time existence by Chow-Tsai [CT1] in the case of expanding convex plane curves, Chow-Tsai [CT2] to remove the homogeneity assumption on the speed of the flow in the theorems of Gerhardt [G], Huisken [H] and Urbas [U1] for expanding convex hypersurfaces, and Chow-Liou-Tsai [CLT] to the equation $u_{t}=F(\Delta u+n u)$ on $S^{n}$, where $F$ is an arbitrary increasing function. Finally, see Tsai $[\mathrm{T}]$ for the global existence and convergence of expanding flows of starshaped plane curves with arbitrary nonhomogeneous speeds. Tsai proved a uniform gradient estimate for the radial function via intricate maximum principle arguments. Using different methods we partially gener- 
alize Tsai's gradient estimate to higher dimensions. Our estimate is weaker than his since it only holds outside a fixed set.

In the second part we consider degenerate parabolic conformal flows of locally conformally flat metrics. We prove a uniform gradient estimate for the logarithm of the conformal factor. This result extends Ye's gradient estimate for the Yamabe flow [Y]. It is easy to see that Ye's proof extends verbatim to strictly parabolic conformal flows since one can apply the strong maximum principle and the Hopf boundary point lemma in this case. However, for degenerate parabolic flows, such a proof is not possible. To circumvent this difficulty, we apply a fixed plane reflection method directly on $S^{n}$, which only uses the weak maximum principle. This version of the Aleksandrov reflection method is analogous to the one used in ChowGulliver [CG1] for flows of convex hypersurfaces and also has the advantage that one can see geometrically how the gradient estimate for the logarithm of the conformal factor arises. For applications to long-time existence and convergence theorems for nonlinear conformal flows, see Liou [L].

\section{Embedded and starshaped hypersurfaces.}

Let $M^{n} \subset \mathbb{R}^{n+1}$ be an embedded smooth hypersurface anc. $\Pi$ be a hyperplane perpendicular to a unit vector $V \in \mathbb{R}^{n+1}$, i.e., there is a constant $C$ such that $\langle\Pi, V\rangle=C$. Denote by $H_{+}(\Pi)=\left\{x \in \mathbb{R}^{n+1}:\langle x, V\rangle>C\right\}$ and $H_{-}(\Pi)=\left\{x \in \mathbb{R}^{n+1}:\langle x, V\rangle<C\right\}$ the half-spaces to both sides of $\Pi$. Let $M^{\Pi}$ be the reflection of $M^{n}$ about the plane $\Pi$, i.e., $M^{\Pi}=$ $\left\{x-2(\langle x, V\rangle-C) V: x \in M^{n}\right\}$.

Definition 2.1. We say that we can

(1) reflect $M^{n}$ strictly at $(\Pi, V)$ if $M^{\Pi} \cap H_{-}(\Pi) \subset \operatorname{int}\left(M^{n}\right) \cap H_{-}$(ח) and $V \notin T M_{x}^{n}$ for any $x \in M^{n} \cap \Pi$. Here $\operatorname{int}\left(M^{n}\right)$ denotes the region interior to $M^{n}$.

(2) reflect $M^{n}$ strictly up to $(\Pi, V)$ if we can reflect $M^{n}$ strictly at; $\left(\Pi^{\prime}, V\right)$, where $\Pi^{\prime}$ is any hyperplane parallel to $\Pi$ such that $\left\langle\Pi^{\prime}, V\right\rangle \geq C$. In particular, this implies $V \notin T M_{x}^{n}$ for any $x \in M^{n} \cap \overline{H_{+}(\Pi)}$.

Let $P^{n}$ be a closed n-dimensional differentiable manifold and $X_{\circ}: P^{n} \rightarrow$ $\mathbb{R}^{n+1}$ parametrize a smooth embedded hypersurface $M_{o}=X_{o}\left(P^{n}\right)$. Suppose that

$$
X: P^{n} \times[0, T) \rightarrow \mathbb{R}^{n+1}
$$


Geometric Aspects of Aleksandrov Reflection and Gradient Estimates 391

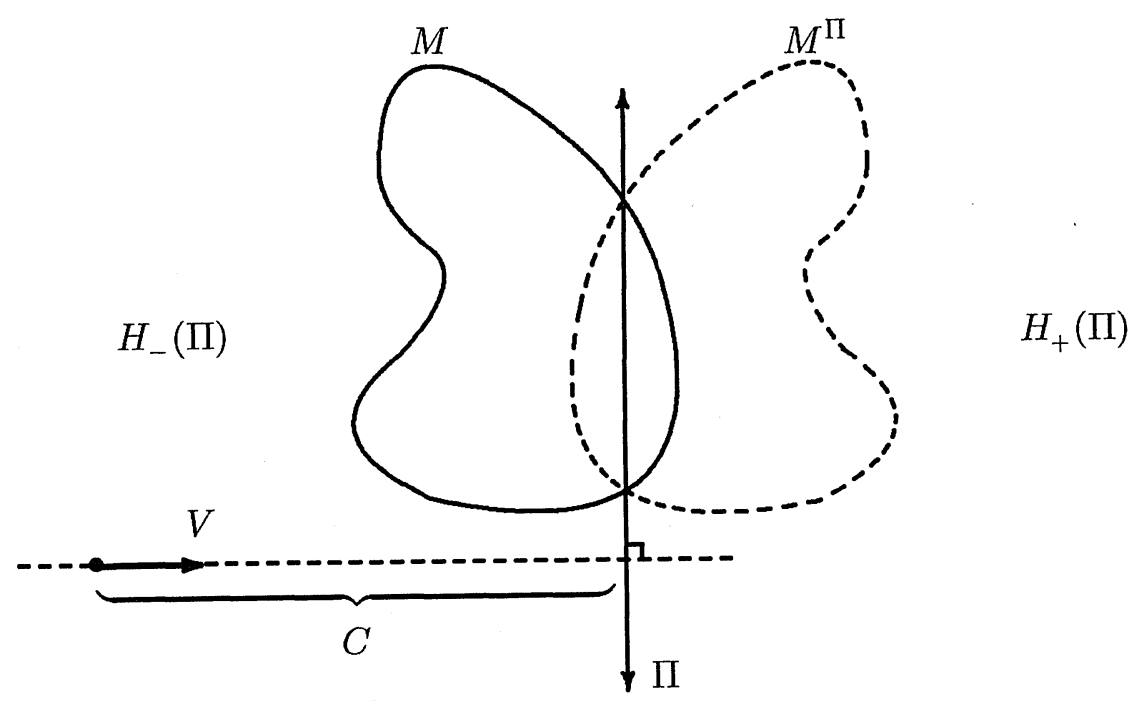

Figure 1. $M^{n}$ reflects strictly at $(\Pi, V)$.

is a solution to the evolution equation

$$
\begin{aligned}
\frac{\partial X}{\partial t} & =F\left(\kappa_{1}, \kappa_{2}, \ldots, \kappa_{n}\right) N \\
X(0) & =X_{o},
\end{aligned}
$$

where $\kappa_{1} \leq \kappa_{2} \leq \cdots \leq \kappa_{n}$ are the principal curvatures in increasing order and $N$ is the unit inward normal to $M_{t}:=X_{t}\left(P^{n}\right)$. Here we assume $F$ : $A \rightarrow \mathbb{R}$ is a $C^{1}$ function, where $A \subset\left\{\left(\lambda_{1}, \lambda_{2}, \ldots, \lambda_{n}\right) \in \mathbb{R}^{n}: \lambda_{1} \leq \lambda_{2} \leq\right.$ $\left.\cdots \leq \lambda_{n}\right\}$, satisfies the strict parabolicity condition: ${ }^{1}$

$$
\frac{\partial F}{\partial \kappa_{i}}>0, \text { for all } 1 \leq i \leq n \text {. }
$$

The main result that we shall apply in this section is the following, which was announced in Chow-Gulliver [CG1]. ${ }^{2}$

Theorem 2.2 (Chow-Gulliver). Let $X: P^{n} \times[0, T) \rightarrow \mathbb{R}^{n+1}$ be an embedded $C^{2}$ solution to equation (2.1)-(2.2), where $F$ satisfies the strict

\footnotetext{
${ }^{1}$ It is not difficult to relax the $C^{1}$ assumption on $F$, assuming a strict monotone property for $F$ as the parabolicity condition.

${ }^{2}$ Extended versions of this theorem will appear in Chow-Gulliver [CG2]. For completeness, we provide a short proof.
} 
parabolicity condition. If we can reflect $M_{o}$ strictly at (resp., up to) (ח,V), then for all $t \in[0, T)$ we can reflect $M_{t}$ strictly at (resp., up to) (I[, $\left.V\right)$.

Proof. We shall show that reflection strictly at $(\Pi, V)$ is preserved under the evolution equation (the preservation of reflection strictly up to $(\Pi, V)$ then follows immediately.) If the result is not true, then there exists $t_{o} \in(0, T)$ such that $M_{t}^{\Pi} \cap H_{-}(\Pi) \subset \operatorname{int}\left(M_{t}\right) \cap H_{-}(\Pi)$ for all $t \in\left[0, t_{o}\right), V \notin T_{x} M_{t}$ for all $x \in M_{t} \cap \Pi$ and $t \in\left[0, t_{o}\right),{ }^{3}$ and either

(i) $M_{t_{o}}^{\Pi} \cap M_{t_{o}} \cap H_{-}(\Pi) \neq \emptyset$ or

(ii) $V \in T_{x_{o}} M_{t_{o}}$, for some $x_{o} \in M_{t_{o}} \bigcap \Pi$.

Case (i): Suppose $x_{o} \in M_{t_{o}}^{\Pi} \cap M_{t_{o}} \cap H_{-}(\Pi)$. In a space-time neighborhood of $\left(x_{o}, t_{o}\right)$ in $\mathbb{R}^{n+1} \times[0, T)$, both $M_{t}^{\Pi} \cap H_{-}(\Pi)$ and $M_{t} \cap H_{-}(\Pi)$ are graphs of smooth functions over open sets in $T_{x_{o}} M_{t_{o}}=T_{x_{o}} M_{t_{o}}^{\Pi}$, which we denote by

$$
f: U \times\left(t_{o}-\epsilon, t_{o}+\epsilon\right) \rightarrow \mathbb{R}
$$

and

$$
f^{\Pi}: U \times\left(t_{o}-\epsilon, t_{o}+\epsilon\right) \rightarrow \mathbb{R},
$$

respectively, where $U \subset T_{x_{o}} M_{t_{o}}$ is an open neighborhood of $x_{o}=0$ in $T_{x_{o}} M_{t_{o}}$. The functions $f$ and $f^{\Pi}$ are uniquely defined by

$$
x+f(x, t) \nu \in M_{t} \quad \text { and } \quad x+f^{\Pi}(x, t) \nu \in M_{t}^{\Pi}
$$

for all $(x, t) \in U \times\left(t_{o}-\epsilon, t_{o}+\epsilon\right)$, where $\nu$ is the unit outward normal to $M_{t_{o}}$ at $x_{o}$. On $U \times\left(t_{o}-\epsilon, t_{o}\right]$, we have $f \geq f^{\Pi}$ and both $f$ and $f^{\Pi}$ are solutions to the same strictly parabolic second order PDE. Since $f\left(0, t_{o}\right)=f^{\Pi}\left(0, t_{o}\right),{ }^{4}$ by the strong maximum principle, we have $f \equiv f^{\Pi}$ on $U \times\left(t_{o}-\epsilon, t_{o}\right]$. This is a contradiction and case (i) is proved.

Case (ii): Suppose $V \in T_{x_{o}} M_{t_{o}}$ for some $x \in M_{t_{o}} \cap \Pi$. We have $T_{x_{o}} M_{t_{o}}^{\Pi}=T_{x_{o}} M_{t_{o}}$ and near $\left(x_{o}, t_{o}\right), M_{t} \cap \overline{H_{-}(\Pi)}$ and $M_{t}^{\Pi} \cap \overline{H_{-}(\overline{\Pi)}}$ are graphs of smooth functions $f$ and $f^{\Pi}: U \times\left(t_{o}-\epsilon, t_{o}+\epsilon\right) \rightarrow \mathbb{R}$, where $U \subset T_{x_{o}} M_{t_{o}} \cap \overline{H_{-}(\Pi)}$ is a half-neighborhood of $x_{o}=0$. Moreover, $f \geq f^{\Pi}$ on $U \times\left(t_{o}-\epsilon, t_{o}\right], V(f)\left(x_{o}, t_{o}\right)=V\left(f^{\Pi}\right)\left(x_{o}, t_{o}\right)$, and $V$ is the unit cutward normal at $x_{o}=0 \in \partial U$. The Hopf boundary point lemma implies $f \equiv f^{\Pi}$ on $U \times\left(t_{o}-\epsilon, t_{o}\right]$, which again is a contradiction and case (ii) is proved.

\footnotetext{
${ }^{3}$ Here $\operatorname{int}\left(M_{t}\right)$ denotes the region interior to $M_{t}$.

${ }^{4}$ Here we identified $x_{o}$ with the origin in $T_{x_{\mathrm{o}}} M_{t_{\mathrm{o}}}$.
} 
Geometric Aspects of Aleksandrov Reflection and Gradient Estimates 393

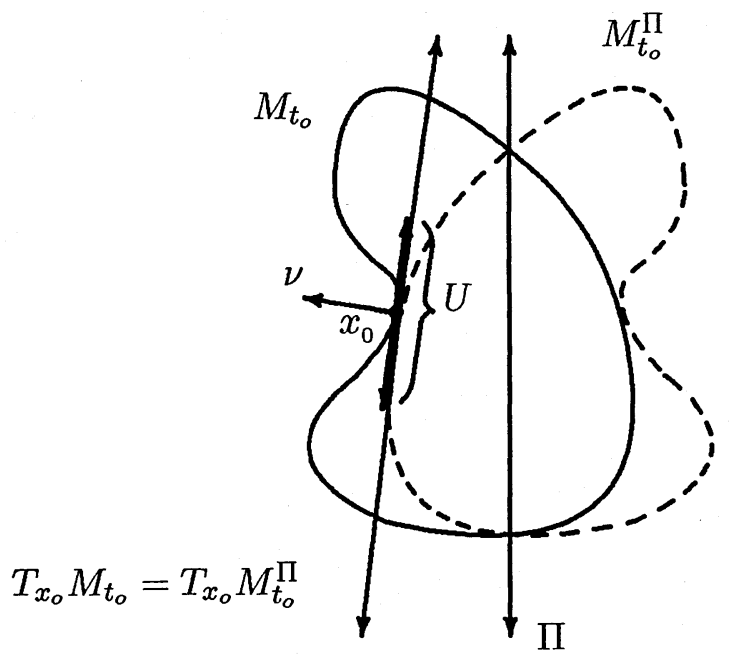

Figure 2. Case (i): interior contact.

Observe that if we can reflect $M^{n}$ strictly up to (ח, $V$ ), then $V \notin T_{x} M^{n}$ for all $x \in M^{n} \cap \overline{H_{+}(\Pi)}$. This implies $M^{n} \cap H_{+}(\Pi)$ is a graph over $\Pi$. Hence we have the following

Corollary 2.3. Let $X: P^{n} \times[0, T) \rightarrow \mathbb{R}^{n+1}$ be an embedded solution to equation (2.1)-(2.2). If we can reflect $M_{o}$ strictly up to $(\Pi, V)$, then for all $t \in[0, T), V \notin T_{x} M_{t}$ for all $x \in M_{t} \cap \overline{H_{+}(\Pi)}$, and $M_{t} \cap \overline{H_{+}(\Pi)}$ is a graph over $\Pi$.

Perhaps more importantly, this implies that the tangential component of a point on $M_{t}$ is uniformly bounded.

Corollary 2.4. Let $X: P^{n} \times[0, T) \rightarrow \mathbb{R}^{n+1}$ be an embedded solution to equation (2.1)-(2.2). There exists a constant $C$ depending only on $M_{o}$ such that

$$
|x-\langle x, N\rangle N| \leq C
$$

for all $x \in M_{t}, t \in[0, T)$, where $N$ is the unit normal to $M_{t}$ at $x$.

Proof. Choose $C>0$ so that $M_{o} \subset B_{C}(0)$, where $B_{C}(0)$ is the ball of radius $C$ centered at the origin. By Theorem 2.2 , we can reflect $M_{t}$ strictly at (ח, $V$ ) for all $V \in S^{n}$ and $\Pi$ such that $\langle\Pi, V\rangle \geq C$. Hence $V \notin T_{x} M_{t}$ for all $x \in M_{t}$ such that $\langle x, V\rangle \geq C$; this is equivalent to $\langle x, W\rangle\left\langle C\right.$ for all $W \in T_{x} M_{t}$ 


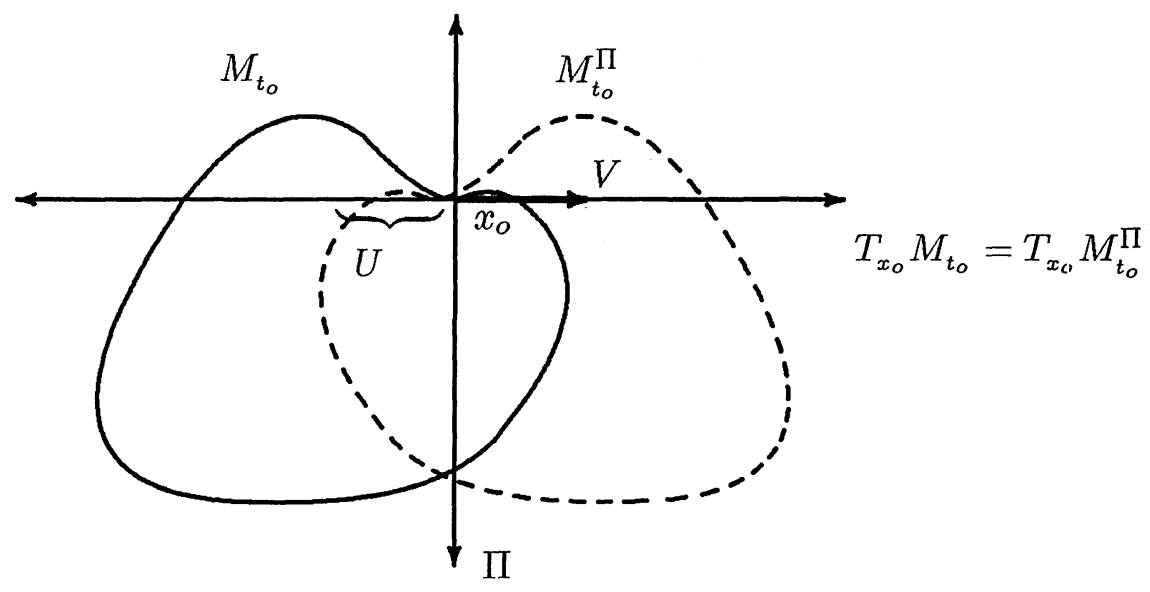

Figure 3. Case (ii): boundary contact.

with $|W|=1$. If we let $W=(x-\langle x, N\rangle N) /|x-\langle x, N\rangle N| \in T_{x} M_{t},{ }^{5}$ then $C>\langle x, W\rangle=|x-\langle x, N\rangle N|$ and the corollary is proved.

In the case where the hypersurfaces $M_{t}$ are convex for all $t \in[0, T)$, this corollary gives a geometric proof of the gradient estimate of ChowGulliver [CG1]. ${ }^{6}$ Recall that the support function $u: S^{n} \rightarrow \mathbb{R}$ of a convex hypersurface $M$ is defined by $u(N)=\langle x, N\rangle$, where $x \in M$ is the unique point with $N$ as its outward unit normal.

Corollary 2.5. Let $u: S^{n} \times[0, T) \rightarrow \mathbb{R}$ be the support functions of the convex hypersurfaces $M_{t}$. There exists a constant $C$ depending only on $u(0)$ such that

$$
|\nabla u(x, t)| \leq C, \quad \text { for all }(x, t) \in S^{n} \times[0, T) .
$$

Proof. Choose the constant $C$ as in the corollary above. Given ariy point $N \in S^{n}$, let $x_{t} \in M_{t}, t \in[0, T)$, be the unique point such that $N$ is the unit outward normal to $M_{t}$ at $x_{t}$. It is a standard formula that $x_{t}=u(N) N+$ $\nabla u(N)$; this implies $\nabla u(N)=x_{t}-\left\langle x_{t}, N\right\rangle N$ and the corollary follows.

When the hypersurfaces $M_{t}$ are starshaped for all $t \in[0, T)$, we obtain a new gradient estimate for the radial function at points outside a certain compact starshaped region associated to the initial hypersurface $M_{o}$. This

\footnotetext{
${ }^{5}$ Of course if $|x-\langle x, N\rangle N|=0$, then we are done.

${ }^{6}$ In Chow-Gulliver [CG1], the gradient estimate is proved under the weaker hypothesis that the equation is degenerate parabolic.
} 
Geometric Aspects of Aleksandrov Reflection and Gradient Estimates 395

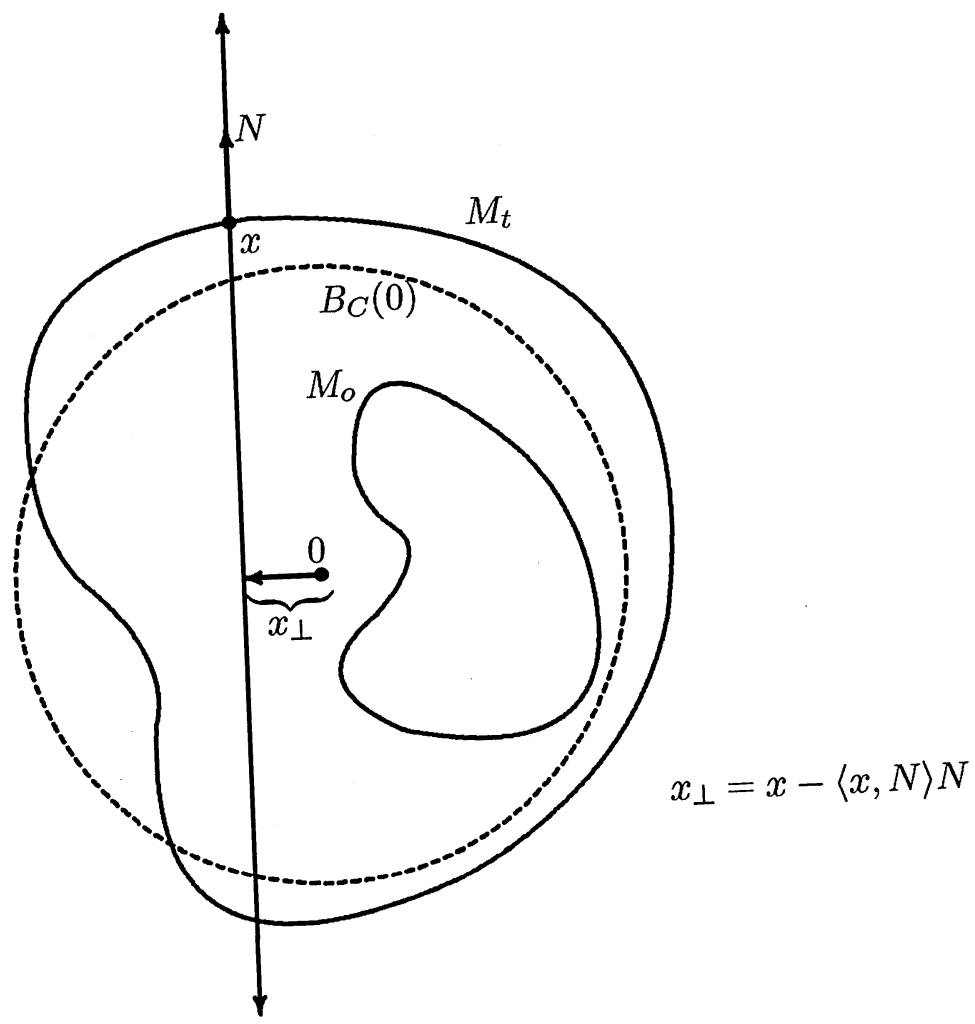

Figure 4. The normal line always intersects $B_{C}(0)$.

gradient estimate relies on the fact that we can reflect up to just inside the interior of the initial hypersurface. ${ }^{7}$ Suppose that $X: P^{n} \times[0, T) \rightarrow \mathbb{R}^{n+1}$ is a solution of (2.1)-(2.2) parametrizing embedded hypersurfaces $M_{t}, t \in[0, T)$. It is clear that for any $V \in S^{n}$, there exists a hyperplane $\Pi$ perpendicular to $V$ such that we can reflect $M_{o}$ up to $(\Pi, V)$ and $\Pi \bigcap \operatorname{int}\left(M_{o}\right) \neq \emptyset$. Actually, we can reflect up to a certain fixed amount in to the interior of $M_{o}$. Given $V \in S^{n}$, there is a unique hyperplane $\Pi_{o}^{V}$ perpendicular to $V$ such that $M_{o} \cap H_{+}\left(\Pi_{o}^{V}\right)=\emptyset$ and $M_{o} \bigcap \Pi_{o}^{V} \neq \emptyset$ (e.g., $\Pi_{o}^{V}=T_{x} M_{o}$ for some $x \in M_{o}$.) By Theorem 2.2 and the compactness of $M_{o}$, we have

Corollary 2.6. Let $X: P^{n} \times[0, T) \rightarrow \mathbb{R}^{n+1}$ be an embedded solution to (2.1)-(2.2). There exists $\epsilon>0$ depending only on $M_{o}$ such that for all $t \in[0, T)$ we can reflect $M_{t}$ up to $\left(\Pi_{o}^{V}-\epsilon V, V\right)$ for every $V \in S^{n}$, where $\Pi_{o}^{V}-\epsilon V=\left\{x-\epsilon V: x \in \Pi_{o}^{V}\right\}$. In particular, if $M_{o} \subset B_{R}(C)$, then we can

\footnotetext{
${ }^{7}$ For this statement we only need the hypersurfaces to be embedded.
} 
reflect $M_{t}$ up to $(\Pi, V)$ provided $H_{+}(\Pi) \cap B_{R-\epsilon}(C)=\emptyset$.

We apply this corollary to starshaped hypersurfaces. Suppose that $X: P^{n} \times$ $[0, T) \rightarrow \mathbb{R}^{n+1}$ is a solution to (2.1)-(2.2) parametrizing hypersurfaces $M_{t}$ starshaped with respect to the origin. The radial function $r: S^{n} \times[0, T) \rightarrow$ $\mathbb{R}_{+}$is defined so that for each $(z, t) \in S^{n} \times[0, T), r(z, t)$ is the unique positive number such that $r(z, t) z \in M_{t}$. We have the following gradient estimate for the radial function.

Proposition 2.7. There exists a constant $C$ depending only on $M_{o}$ such that for all $(z, t) \in S^{n} \times[0, T)$ with $r(z, t) z \in \overline{H_{+}\left(\Pi_{o}^{z}\right)}$, we have ${ }^{8}$

$$
|\nabla r(z, t)| \leq C .
$$

Proof. By Corollary 2.6, there exists a constant $\delta>0$ depending only on $M_{o}$ such that for all $(z, t) \in S^{n} \times[0, T)$ with $r(z, t) z \in \overline{H_{+}\left(\Pi_{o}^{z}\right)}$ and all $W \in S^{n}$ such that $\langle W, z\rangle \geq 1-\delta$, we can reflect $M_{t}$ up to ( $\left.\Pi^{W}, W\right)$, where $\Pi^{W}=\{r(z, t) z+X:\langle X, W\rangle=0\}$. Hence $W \notin T_{r(z, t) z} M_{t}$ for all such $W$, which implies the gradient estimate

$$
|\nabla r(z, t)| \leq \frac{1-\delta}{\sqrt{\delta(2-\delta)}} r
$$

The proposition follows from this estimate and the following

Lemma 2.8. There exists a constant $C$ depending only on $M_{o}$ such that at all points $(z, t) \in S^{n} \times[0, T)$,

$$
r^{2}|\nabla r|^{2} \leq C\left(r^{2}+|\nabla r|^{2}\right) .
$$

In particular, if $r^{2}>C$, then

$$
|\nabla r|^{2} \leq \frac{C r^{2}}{r^{2}-C}
$$

Proof. Substituting the formulas $x=r(z) z$ and

$$
N=\frac{r(z) z-\nabla r(z)}{\sqrt{r(z)^{2}+|\nabla r(z)|^{2}}}
$$

\footnotetext{
${ }^{8}$ This gradient estimate actually holds on a larger set.
} 
Geometric Aspects of Aleksandrov Reflection and Gradient Estimates 397

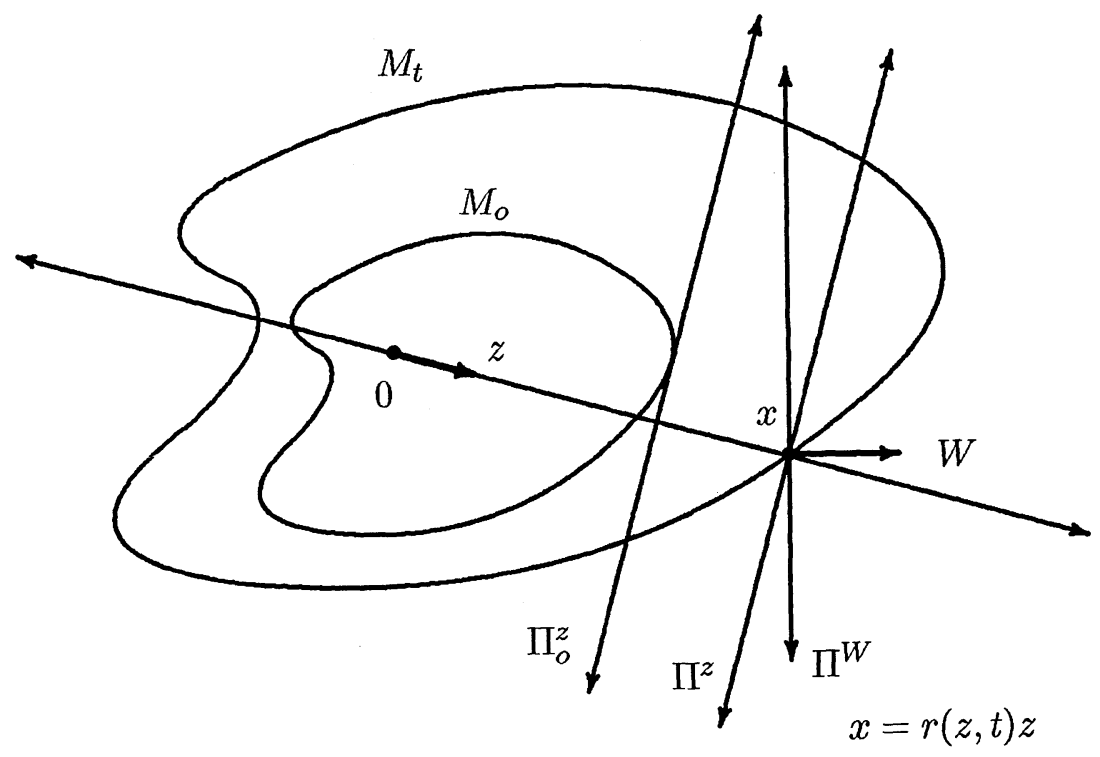

Figure 5. Tilting the plane of reflection.

into Corollary 2.4 yields the inequality

$$
\left|r \cdot z-\frac{r^{2}(r \cdot z-\nabla r)}{r^{2}+|\nabla r|^{2}}\right| \leq C
$$

which is equivalent to

$$
r^{2}|\nabla r|^{4}+r^{4}|\nabla r|^{2} \leq C\left(r^{2}+|\nabla r|^{2}\right)^{2},
$$

and the lemma follows.

Finally, for general embedded solutions, we observe the following easy consequence of the main theorem.

Corollary 2.9. Let $X: P^{n} \times[0, T) \rightarrow \mathbb{R}^{n+1}$ be a smooth embedded solution to equation (2.1)-(2.2), where $F$ satisfies the strict parabolicity condition. Then

(i) there exists a constant $C$ depending only on $M_{o}$ such that

$$
\max _{x \in M_{t}}|x|-\min _{x \in M_{t}}|x| \leq C,
$$

for all $t \in[0, T)$. 


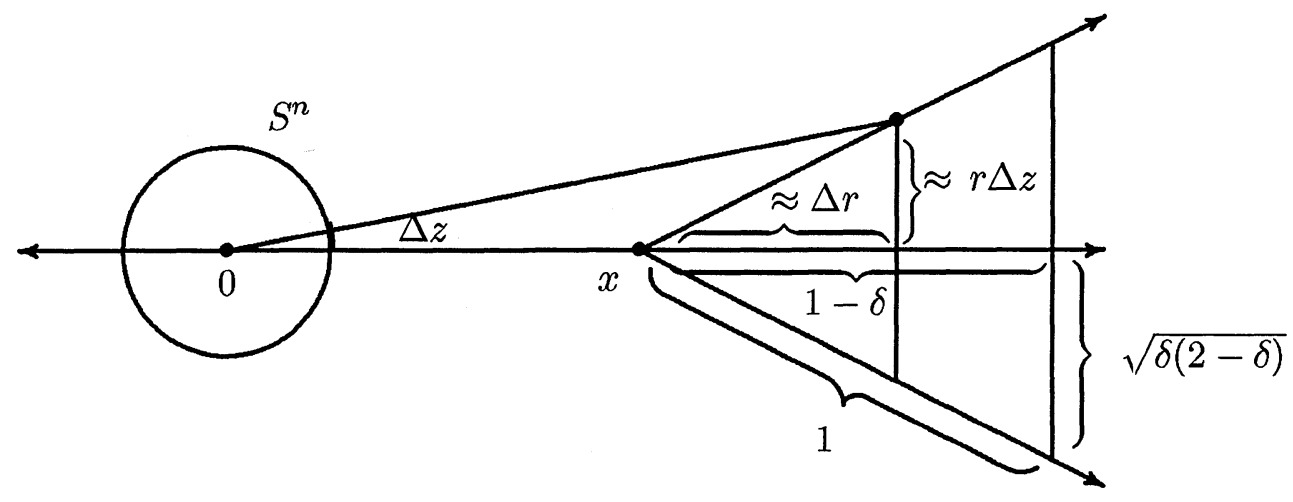

Figure 6. Largest possible gradient: $\Delta r / r \Delta z \approx(1-\delta) / \sqrt{\delta(2-\overline{-}-}$

(ii) there exists a ball $B$ depending only on $M_{o}$ such that $M_{t} \sim B$ is starshaped with respect to the center of $B$ for all $t \in[0, T)$. In purticular, one may take $B$ to be any ball such that $M_{o} \subset \overline{\operatorname{int}(B)}$.

Proof. As before, choose $C$ such that $M_{o} \subset B_{C}(0)$. Given $t \in[0, T)$, choose $x_{1}, x_{2} \in M_{t}$ such that $\left|x_{1}\right|=\min _{x \in M_{t}}|x|$ and $\left|x_{2}\right|=\max _{x \in M_{t}}|x|$. Define the hyperplane $\Pi$ by

$$
\Pi=\left\{W \in \mathbb{R}^{n+1}:\left\langle W, x_{2}-x_{1}\right\rangle=C\left|x_{2}-x_{1}\right|\right\} .
$$

By Theorem 2.2, we can reflect $M_{t}$ strictly up to $\left(\Pi, \frac{x_{2}-x_{1}}{\left|x_{2}-x_{1}\right|}\right)$ for all $t \in$ $[0, T)$. This implies $\operatorname{dist}\left(x_{2}, \Pi\right)<\operatorname{dist}\left(x_{1}, \Pi\right)$, or equivalently

$$
\left\langle x_{2}, \frac{x_{2}-x_{1}}{\left|x_{2}-x_{1}\right|}\right\rangle-C<\left\langle x_{1}, \frac{x_{1}-x_{2}}{\left|x_{2}-x_{1}\right|}\right\rangle+C .
$$

Hence $\left|x_{2}\right|^{2}-\left|x_{1}\right|^{2}<2 C\left|x_{2}-x_{1}\right| \leq 4 C\left|x_{2}\right|$, and thus $\left|x_{2}\right| \leq\left|x_{1}\right|+4 C$.

(ii): This follows from Corollary 2.3, we leave the details of the proof to the reader.

\section{Conformal flows on $S^{n}$.}

In this section we consider degenerate parabolic second order conformal flows invariant under the conformal diffeomorphism group of $S^{n}$. As 


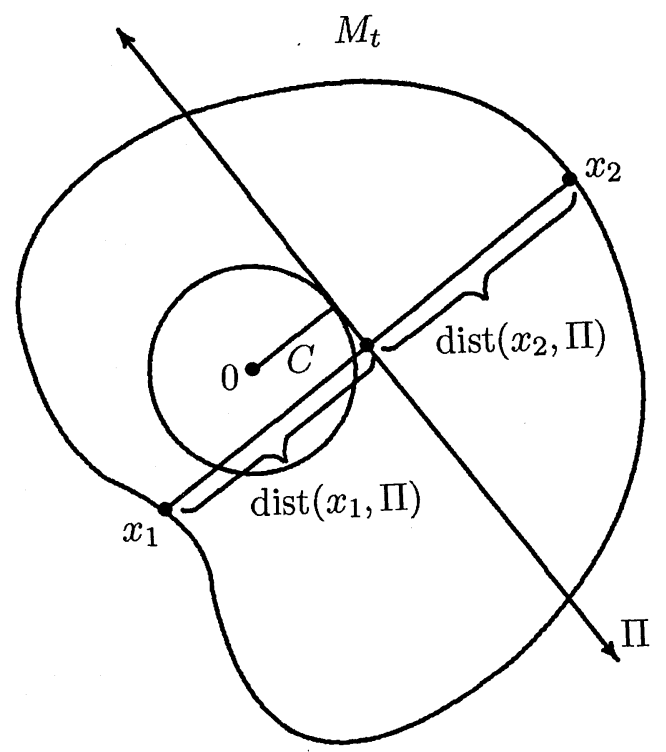

Figure 7. $M_{t}$ reflects strictly up to $\left(\Pi, \frac{x_{2}-x_{1}}{\left|x_{2}-x_{1}\right|}\right)$.

remarked in the introduction, it is easy to see that Ye's proof of the gradient estimate for the conformal factor of the metric under the Yamabe flow $[\mathrm{Y}]$ extends to uniformly parabolic flows since the strong maximum principle and the Hopf boundary point lemma may be used. We observe that a fixed plane reflection method applies to conformal flows. This method is essentially equivalent to the Aleksandrov moving plane method pioneered by Gidas-Ni-Nirenberg [GNN], Schoen [S1],[S2] for scalar curvature problems, and Ye's extension to the Yamabe flow. However, for the parabolic flow our fixed plane method has the advantage that only the weak maximum principle is used, which allows for degenerate parabolic flows. We also apply the reflection method directly on $S^{n}$ instead of the usual practice of stereographically projecting the equation to $\mathbb{R}^{n}$. This makes Ye's gradient estimate (and our extension) more transparent geometrically.

Let $N \in S^{n} \subset \mathbb{R}^{n+1}$ be an arbitrary point, which we shall consider as the north pole, that is, we rotate coordinates on $\mathbb{R}^{n+1}$ so that $N=(0, \ldots, 0,1)$. The south pole shall be denoted by $S=-N$. Stereographic projection

$$
\sigma: S^{n}-N \rightarrow \mathbb{R}^{n}
$$

is defined by

$$
\sigma\left(y_{1}, \ldots, y_{n+1}\right)=\left(\frac{y_{1}}{1-y_{n+1}}, \ldots, \frac{y_{n}}{1-y_{n+1}}\right),
$$


and its inverse is given by

$$
\sigma^{-1}\left(x_{1}, \ldots, x_{n}\right)=\left(\frac{2 x_{1}}{|x|^{2}+1}, \ldots, \frac{2 x_{n}}{|x|^{2}+1}, \frac{|x|^{2}-1}{|x|^{2}+1}\right) .
$$

We also have the reflection about the equator

$$
\rho: S^{n} \rightarrow S^{n}
$$

defined by

$$
\rho(y)=y-2\langle y, N\rangle N .
$$

Finally, we introduce the conformal diffeomorphisms corresponding via $\sigma$ to dilations in $\mathbb{R}^{n}$. Given $\alpha>0$, define

$$
\varphi_{\alpha}=\sigma^{-1} \circ \alpha \circ \sigma: S^{n} \rightarrow S^{n},
$$

where $\alpha: \mathbb{R}^{n} \rightarrow \mathbb{R}^{n}$ is multiplication by $\alpha$. In particular, $\varphi_{\alpha}$ fixes both $N$ and $S$, and $\varphi_{1}$ is the identity. Moreover, $\varphi_{\alpha}$ takes points closer to $S$ for $\alpha<1$, and takes points closer to $N$ for $\alpha>1$. Hence, for any metric $g$ on $S^{n}, \varphi_{\alpha}^{*} g$ is concentrated more near $N$ for $\alpha<1$, and concentrated more near $S$ for $\alpha>1$.

We now consider an arbitrary metric $g$ conformal to $g_{S^{n}}$. Let $u: S^{n} \rightarrow \mathbb{R}$ be the conformal factor defined by

$$
g=e^{u} g_{S^{n}} .
$$

It is somewhat clear geometrically that if we concentrate the metric $g$ enough near $N$ by pulling back by $\varphi_{\alpha}$, it will be greater than its reflection on the northern hemisphere. However, to prove this in detail requires a computation, which we shall carry out below. Let $S_{N}^{n}=\left\{y \in S^{n}:\left\langle y, N_{i} \geq 0\right\}\right.$ denote the northern hemisphere.

Proposition 3.1. There exists a constant $\alpha_{o} \in(0,1)$ such that for all $\alpha \in$ $\left(0, \alpha_{o}\right]$

$$
\varphi_{\alpha}^{*} g \geq \rho^{*} \varphi_{\alpha}^{*} g \quad \text { on } S_{N}^{n} .
$$

Moreover, $\alpha_{o}$ may be chosen independent of $N \in S^{n}$.

Proof. The formula for the pull-back by $\sigma^{-1}$ to $\mathbb{R}^{n}$ of an arbitrary metric conformally related to $g_{S^{n}}$ is

$$
\left(\sigma^{-1}\right)^{*}\left(e^{u} g_{S^{n}}\right)(x)=\frac{4}{\left(|x|^{2}+1\right)^{2}} e^{u \circ \sigma^{-1}(x)} g_{R^{n}}(x), \quad x \in \mathbb{R}^{n} .
$$


Geometric Aspects of Aleksandrov Reflection and Gradient Estimates 401

Using the formula $|\sigma(y)|^{2}=\frac{1+y_{n+1}}{1-y_{n+1}}$, we compute that the pull-back by $\sigma$ to $S^{n}$ of an arbitrary metric conformally related to $g_{R^{n}}$ is given by

$$
\sigma^{*}\left(e^{v} g_{R^{n}}\right)(y)=\frac{1}{\left(1-y_{n+1}\right)^{2}} e^{v \circ \sigma(y)} g_{S^{n}}(y), \quad y \in S^{n} .
$$

Finally, we observe that

$$
\alpha^{*}\left(e^{w} g_{R^{n}}\right)(x)=\alpha^{2} e^{w(\alpha x)} g_{R^{n}}(x) .
$$

Combining all of the considerations above, we find that

$$
\varphi_{\alpha}^{*} g(y)=\sigma^{*} \alpha^{*}\left(\sigma^{-1}\right)^{*}\left(e^{u} g_{S^{n}}\right)(y)=\frac{4 \alpha^{2}}{\left[\alpha^{2}+1-\left(1-\alpha^{2}\right) y_{n+1}\right]^{2}} e^{u\left(\varphi_{\alpha}(y)\right)} g_{S^{n}}(y) .
$$

By the formula above, the proposition is equivalent to showing that for $\alpha$ sufficiently small,

$$
\frac{\alpha^{2}+1+\left(1-\alpha^{2}\right) y_{n+1}}{\alpha^{2}+1-\left(1-\alpha^{2}\right) y_{n+1}} \geq e^{\frac{1}{2}\left[u\left(\varphi_{\alpha} \circ \rho(y)\right)-u\left(\varphi_{\alpha}(y)\right)\right]},
$$

for all $y \in S_{N}^{n}$. First observe that using the formula above and the inequality

$$
\operatorname{osc}_{S^{n}} u:=\max _{S^{n}} u-\min _{S^{n}} u \geq u\left(\varphi_{\alpha} \circ \rho(y)\right)-u\left(\varphi_{\alpha}(y)\right),
$$

we find that if $y \in S^{n}$ satisfies

$$
y_{n+1} \geq \frac{e^{\mathrm{osc}_{S^{n}} u / 2}-1}{e^{\mathrm{OSc}_{S^{n}} u / 2}+1} \cdot \frac{\alpha^{2}+1}{1-\alpha^{2}},
$$

then $\varphi_{\alpha}^{*} g(y) \geq \rho^{*} \varphi_{\alpha}^{*} g(y)$. In particular, if $\alpha \leq e^{-\operatorname{Osc}_{S^{n}} u / 2}$, then for all $y \in S^{n}$ such that

$$
y_{n+1} \geq \frac{1+e^{\mathrm{OSc}_{S^{n}} u}}{\left(1+e^{\mathrm{OSc}_{S^{n}} u / 2}\right)^{2}}
$$

we have $\varphi_{\alpha}^{*} g(y) \geq \rho^{*} \varphi_{\alpha}^{*} g(y)$. That is, for $\alpha$ sufficiently small and $y$ sufficiently close to $N$ (depending only on $g$ ), the metric is greater than or equal to its reflection.

On the other hand, using the previous formulas for $\sigma, \sigma^{-1}$, and $\alpha$, we compute that

$$
\varphi_{\alpha}(y)_{n+1}=\frac{\alpha^{2}-1+\left(\alpha^{2}+1\right) y_{n+1}}{\alpha^{2}+1+\left(\alpha^{2}-1\right) y_{n+1}}, \quad y \in S^{n} .
$$


Let $L=\sup _{x \neq y}|u(x)-u(y)| / \operatorname{dist}_{S^{n}}(x, y)$ be the Lipschitz constant of $u$ on $S^{n}$. To prove the proposition, it is sufficient to show that for any $\epsilon>0$, there exists $\alpha_{o} \in(0,1)$ such that for all $\alpha \in\left(0, \alpha_{o}\right]$, we have

$$
\frac{\alpha^{2}+1+\left(1-\alpha^{2}\right) y_{n+1}}{\alpha^{2}+1-\left(1-\alpha^{2}\right) y_{n+1}} \geq e^{\frac{L}{2} \operatorname{dist}_{S^{n}}\left(\varphi_{\alpha} \circ \rho(y), \varphi_{\alpha}(y)\right)}
$$

for all $y \in S_{N}^{n}$ with $y_{n+1} \leq 1-\epsilon$, where

$$
\begin{aligned}
\operatorname{dist}_{S^{n}}\left(\varphi_{\alpha} \circ \rho(y), \varphi_{\alpha}(y)\right)= & \sin ^{-1}\left(\frac{\alpha^{2}-1+\left(\alpha^{2}+1\right) y_{n+1}}{\alpha^{2}+1+\left(\alpha^{2}-1\right) y_{n+1}}\right) \\
& -\sin ^{-1}\left(\frac{\alpha^{2}-1-\left(\alpha^{2}+1\right) y_{n+1}}{\alpha^{2}+1-\left(\alpha^{2}-1\right) y_{n+1}}\right) .
\end{aligned}
$$

If we let $z=y_{n+1}, \beta=\alpha^{2}$, and $C=L / 2$, then the inequality above is equivalent to showing that for all $\epsilon>0$ there exists $\beta_{o} \in(0,1)$ such that for all $\beta \in\left(0, \beta_{o}\right]$ and $z \in[0,1-\epsilon]$

$$
\Phi(\beta, z) \leq 1
$$

where

$$
\begin{gathered}
\Phi(\beta, z)=\frac{\beta+1-(1-\beta) z}{\beta+1+(1-\beta) z} \exp \left\{C \left[\sin ^{-1}\left(\frac{\beta-1+(\beta+1) z}{\beta+1+(\beta-1) z}\right)\right.\right. \\
\left.\left.-\sin ^{-1}\left(\frac{\beta-1-(\beta+1) z}{\beta+1-(\beta-1) z}\right)\right]\right\} .
\end{gathered}
$$

Observe that $\Phi:(0,1] \times[0,1] \rightarrow \mathbb{R}$ is differentiable and $\Phi(\beta, 0)=1$. One computes that ${ }^{9}$

$$
\frac{\partial}{\partial z} \ln \Phi(\beta, z)=\left(\beta-1+\frac{2 C \sqrt{\beta}}{\sqrt{1-z^{2}}}\right) \frac{2(1+\beta)}{(1+\beta+z-\beta z)(1+\beta-z+\beta z)} .
$$

Hence, we have

$$
\frac{\partial}{\partial z} \ln \Phi(\beta, z) \leq 0
$$

if and only if

$$
\beta-1+\frac{2 C \sqrt{\beta}}{\sqrt{1-z^{2}}} \leq 0
$$

\footnotetext{
${ }^{9}$ We first computed this using mathematica and subsequently easily verified the formula by hand.
} 
Geometric Aspects of Aleksandrov Reflection and Gradient Estimates 403

which is equivalent to

$$
z \leq \sqrt{1-\frac{4 C^{2} \beta}{(1-\beta)^{2}}} .
$$

Since $\Phi(\beta, 0)=1$, we have $\Phi(\beta, z) \leq 1$ for all $(\beta, z)$ such that $z \leq$ $\sqrt{1-\frac{4 C^{2} \beta}{(1-\beta)^{2}}}$. Combining this with our previous inequality, we obtain that $\Phi(\beta, z) \leq 1$ for all $(\beta, z)$ such that $\beta$ is sufficiently small. The proposition follows.

Let $u_{\alpha}: S^{n} \rightarrow \mathbb{R}$ denote the conformal factor of the metric $\varphi_{\alpha}^{*} g$ :

$$
\varphi_{\alpha}^{*} g=e^{u_{\alpha}} g_{S^{n}} .
$$

Clearly

$$
\rho^{*} \varphi_{\alpha}^{*} g=e^{u_{\alpha} \circ \rho} g_{S^{n}} .
$$

The proposition may then be rephrased as saying

$$
u_{\alpha} \geq u_{\alpha} \circ \rho \quad \text { on } S_{N}^{n}
$$

for all $\alpha \in\left(0, \alpha_{o}\right]$. Let $\partial S_{N}^{n}=\left\{y \in S^{n}:\langle y, N\rangle=0\right\}$ denote the equator. We then have for all $y \in \partial S_{N}^{n}$,

$$
\left\langle\nabla u_{\alpha}(y), N\right\rangle \geq 0,
$$

noting that $N \in T S_{y}^{n}$. By the formula for $\varphi_{\alpha}^{*} g(y)$, we have

$$
u_{\alpha}(y)=u \circ \varphi_{\alpha}(y)+\ln \frac{4 \alpha^{2}}{\left[\alpha^{2}+1-\left(1-\alpha^{2}\right) y_{n+1}\right]^{2}}, \quad y \in S^{n} .
$$

Hence

$$
\left\langle\nabla u_{\alpha}(y), N\right\rangle=\left\langle\nabla\left(u \circ \varphi_{\alpha}\right)(y), N\right\rangle+\frac{2\left(1-\alpha^{2}\right)\left\langle\nabla y_{n+1}, N\right\rangle}{\alpha^{2}+1-\left(1-\alpha^{2}\right) y_{n+1}} .
$$

Extend the vector field $N$, defined on the equator, to a vector field $\bar{N}$ on $S^{n} \sim\{N, S\}$ by parallel translating $N$ along great circles passing through $N$ and $S$. By the formula above, we have for $y \in \partial S_{N}^{n}$ (the equator),

$$
\left\langle\nabla u_{\alpha}(y), N\right\rangle=\frac{2 \alpha}{\alpha^{2}+1}\left\langle\nabla u\left(\varphi_{\alpha}(y)\right), \bar{N}\right\rangle+\frac{2\left(1-\alpha^{2}\right)}{\alpha^{2}+1},
$$

where we used $y_{n+1}=0,\left\langle\nabla y_{n+1}, N\right\rangle=1$, and $\left|\left(\varphi_{\alpha}\right)_{*} V\right|=\frac{2 \alpha}{\alpha^{2}+1}|V|$ for all $V \in T S_{y}^{n}$. Since $\left\langle\nabla u_{\alpha}(y), N\right\rangle \geq 0$, we have

$$
\left\langle\nabla u\left(\varphi_{\alpha}(y)\right), \bar{N}\right\rangle \geq-\frac{1-\alpha^{2}}{\alpha}
$$




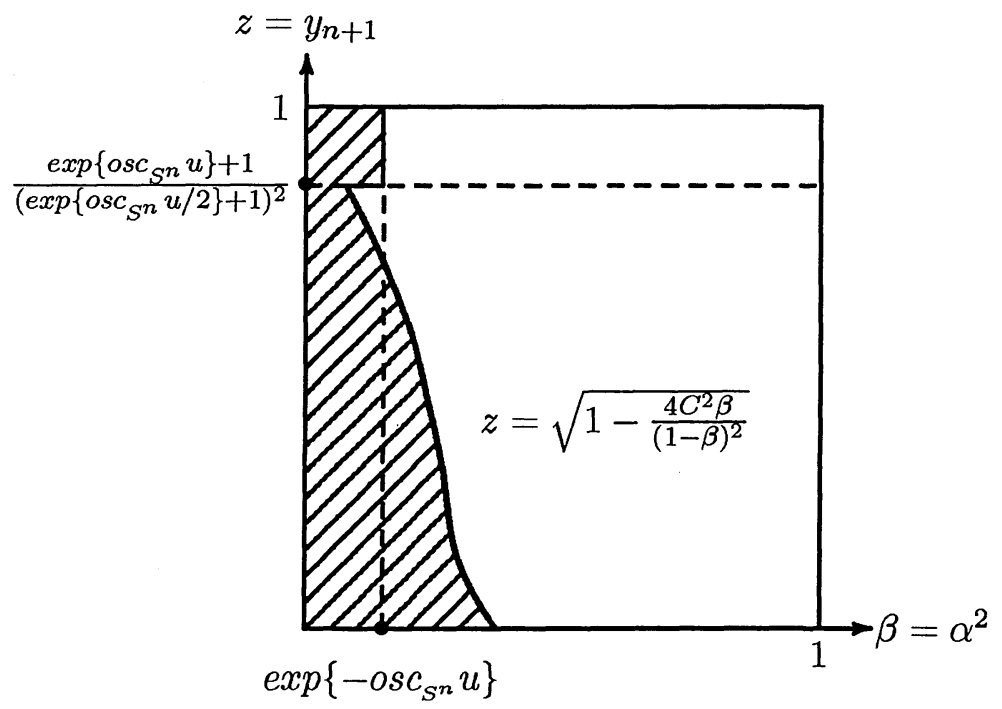

Figure 8. $\Phi(\beta, z) \leq 1$ in the shaded region.

for all $\alpha \in\left(0, \alpha_{o}\right]$. Noting that the only assumption on $y$ and $N$ is $\langle y, N\rangle=0$, we conclude that for every $y, z \in S^{n}$ such that $\langle y, z\rangle=0$,

$$
\left\langle\nabla u\left(\varphi_{\alpha_{o}}^{z}(y)\right), z\right\rangle \geq-\frac{1-\alpha_{o}^{2}}{\alpha_{o}},
$$

where $\varphi_{\alpha_{o}}^{z}=\varphi_{\alpha_{o}}$ is the conformal diffeomorphism as before except with $z$ as the north pole, and $z$ is extended to a vector field $\bar{z}$ on $S^{n} \sim\{z,-z\}$ in the same way we extended $N$ to $\bar{N}$ on $S^{n} \sim\{N, S\}$.

Now given any $x \in S^{n}$, let

$$
E_{\alpha_{o}}(x)=\left\{y \in S^{n}: \varphi_{\alpha_{o}}^{z}(y)=x \text { for some } z \in S^{n} \text { with }\langle y, z\rangle=0\right\} .
$$

By equation (3.1) we have $\left\langle\varphi_{\alpha_{o}}^{z}(y), z\right\rangle=\left(\alpha_{o}^{2}-1\right) /\left(\alpha_{o}^{2}+1\right)$ for all $y \in S^{n}$ such that $\langle y, z\rangle=0$. This implies

$$
E_{\alpha_{o}}(x)=\left\{y \in S^{n}:\langle y, x\rangle=\frac{2 \alpha_{o}}{\alpha_{o}^{2}+1}\right\},
$$

which is an (n-1)-sphere centered at $x$, and

$$
\begin{aligned}
F_{\alpha_{o}}(x) & :=\left\{z \in S^{n}: \varphi_{\alpha_{o}}^{z}(y)=x \text { for some } y \in S^{n} \text { with }\langle y, z\rangle=0\right\} \\
& =\left\{z \in S^{n}:\langle z, x\rangle=\frac{\alpha_{o}^{2}-1}{\alpha_{o}^{2}+1}\right\}
\end{aligned}
$$




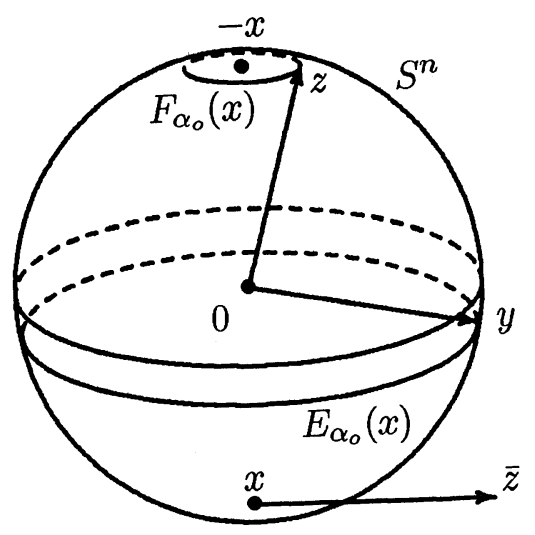

Figure 9. The (n-1)-spheres $E_{\alpha_{o}}(x)$ and $F_{\alpha_{o}}(x)$.

Since $F_{\alpha_{o}}(x)$ is an (n-1)-sphere, taking $x=\varphi_{\alpha_{o}}^{z}(y)$ fixed in equation (3.2) implies $\langle\nabla u(x), \bar{z}\rangle \geq-\left(1-\alpha_{o}^{2}\right) / \alpha_{o}$ for all $\bar{z} \in S^{n}$ such that $\langle\bar{z}, x\rangle=0$. Therefore

$$
|\nabla u(x)| \leq \frac{1-\alpha_{o}^{2}}{\alpha_{o}}, \quad x \in S^{n} .
$$

Now the discussion above may seem trivial since $u$ is any function on $S^{n}$ and the constant $\alpha_{o}$ depends on $u$. However, the key point is this. Suppose that $g=e^{u} g_{S^{n}}$ is a solution to a parabolic second order conformal flow, invariant under the (conformal) diffeomorphism group of $S^{n}$. By the (weak) maximum principle, if $\left(\varphi_{\alpha}^{z}\right)^{*} g \geq \rho^{*}\left(\varphi_{\alpha}^{z}\right)^{*} g$ on $S_{z}^{n}:=\left\{x \in S^{n}:\langle x, z\rangle \geq 0\right\}$ at $t=0$, then it remains so for $t>0$. Hence, by our previous remarks, we have a uniform gradient estimate for the conformal factor $u$. We formulate this result in more detail below.

Let $F: S^{2} T^{*} S^{n} \oplus \mathbb{R}_{+} g_{S^{n}} \times[0, T) \rightarrow \mathbb{R}$, where $S^{2} T^{*} S^{n}$ denotes the bundle of symmetric covariant 2-tensors on $S^{n}$ and $\mathbb{R}_{+} g_{S^{n}}$ denotes the positive ray in the subbundle $\mathbb{R} g_{S^{n}}=\left\{u \cdot g_{S^{n}}(x): u \in \mathbb{R}, x \in S^{n}\right\} \subset S^{2} T^{*} S^{n}$, be a function such that $F(\beta(x), \gamma(x), t)$ depends only on the eigenvalues of the symmetric 2-tensor $\beta$ with respect to the metric $\gamma$ and the time $t$. We consider the evolution equation

$$
\begin{aligned}
\frac{\partial g}{\partial t}(x, t) & =F\left(\operatorname{Ric}_{g(t)}(x), g(x, t), t\right) \cdot g(x, t), \quad x \in S^{n}, t \in[0, T) \\
g(0) & =g_{o}=e^{u_{o}} g_{S^{n}}
\end{aligned}
$$


where $\operatorname{Ric}_{g(t)}$ denotes the Ricci tensor of the metric $g(t)$. Equation (3.3) is invariant under the group of conformal diffeomorphisms, that is, if $g(t)$ is a solution to (3.3) and $\psi$ is a conformal diffeomorphism of $S^{n}$, then $\psi^{*}(g(t))$ is also a solution of equation (3.3). We shall also assume that equation (3.3) is (degenerate) parabolic, that is, $F$ satisfies the following monotone property: $F\left(\beta_{1}-\frac{1}{2(n-1)} \operatorname{tr}_{\gamma}\left(\beta_{1}\right) \gamma, \gamma, t\right) \geq F\left(\beta_{2}-\frac{1}{2(n-1)} \operatorname{tr}_{\gamma}\left(\beta_{2}\right) \gamma, \gamma, t\right)$ for all $t \in[0, T), x \in S^{n}, \gamma \in \mathbb{R}_{+} g_{S^{n}}(x)$, and $\beta_{1}, \beta_{2} \in S^{2} T^{*} S_{x}^{n}$ such that $\beta_{1} \leq \beta_{2}$. When $n \geq 3$, this condition arises because if $\tilde{g}_{i j}=f g_{i j}$ is a conformal variation of $g_{i j}$, then $\left(R_{i j}-\frac{R}{2(n-1)} g_{i j}\right)^{\sim}=-\frac{n-2}{2} \nabla_{i} \nabla_{j} f$ is the variation of $R_{i j}-\frac{R}{2(n-1)} g_{i j}$. When $n=2$ we assume instead that $F$ is a nonincreasing function of $R$. Degenerate parabolicity of our equation will ensure that we can apply an Aleksandrov-type reflection argument to the solution $g$. We have the following generalization of Ye's gradient estimate for the Yamabe flow.

Theorem 3.2. If $g(t)=e^{u(t)} g_{S^{n}}, t \in[0, T)$, is a solution to equation (3.3), then there exists a constant $C$ depending only on $g(0)$ (and not on $F$ ), such that

$$
|\nabla u(x, t)| \leq C, \quad \text { for all } x \in S^{n}, t \in[0, T) .
$$

Proof. By Proposition 3.1, there exists a constant $\alpha_{o} \in(0,1)$ such that for all $z \in S^{n}$ and $\alpha \in\left(0, \alpha_{o}\right]$,

$$
\left(\varphi_{\alpha}^{z}\right)^{*} g(0) \geq \rho^{*}\left(\varphi_{\alpha}^{z}\right)^{*} g(0) \quad \text { on } S_{z}^{n},
$$

where $S_{z}^{n}=\left\{y \in S^{n}:\langle y, z\rangle \geq 0\right\}$. By the conformal invariance of equation (3.3), both $\left(\varphi_{\alpha}^{z}\right)^{*} g(t)$ and $\rho^{*}\left(\varphi_{\alpha}^{z}\right)^{*} g(t)$ are solutions to the same degenerate parabolic equation. Hence, by the weak maximum principle, we have $\left(\varphi_{\alpha}^{z}\right)^{*} g(t) \geq \rho^{*}\left(\varphi_{\alpha}^{z}\right)^{*} g(t)$ on $S_{z}^{n}$ for all $t \in[0, T)$ and $\alpha \in\left(0, \alpha_{o}\right]$. By our previous remarks, this implies the uniform gradient estimate

$$
|\nabla u(x, t)| \leq \frac{1-\alpha_{o}^{2}}{\alpha_{o}}, \quad \text { for all } x \in S^{n}, t \in[0, T) .
$$

As in $[Y]$, we can extend this theorem to conformal flows of metrics on a domain $\Omega$ in $S^{n}$, provided the logarithm of the conformal factor tends to infinity at $\partial \Omega$ on $[0, T)$. In particular, we obtain a uniform gradient estimate for $u$ on $K \times[0, T)$, for any compact subset $K \subset \Omega$, depending only on $K$ and the initial metric. Using the developing map theorem of Schoen-Yau [SY], this yields a uniform gradient estimate for the logarithm 
Geometric Aspects of Aleksandrov Reflection and Gradient Estimates 407

of the conformal factor for degenerate parabolic conformal flows on compact locally conformally flat manifolds.

Theorem 3.3. Let $g(x, t)=e^{u(x, t)} g_{S^{n}}(x)$ be a solution to equation (3.3) on $\Omega \times[0, T)$ such that $\lim _{x \rightarrow \partial \Omega} u(x, t)=\infty$ for all $t \in[0, T)$. For any compact subset $K \subset \Omega$, there exists a constant $C$ depending only on $K$ and $g_{o}$ such that

$$
|\nabla u(x, t)| \leq C
$$

for all $x \in K$ and $t \in[0, T)$.

Before proving the theorem, we make some preliminary observations. Given $x \in S^{n}$ and $\rho \in(0, \pi / 2)$, the conformal diffeomorphism (with $-x$ as the north pole) $\varphi_{\alpha}^{-x}: S^{n} \rightarrow S^{n}$ maps the southern hemisphere $\left\{y \in S^{n}\right.$ : $\langle y, x\rangle \geq 0\}$ onto $B_{\rho}(x)$ if and only if $\alpha=\frac{1-\cos \rho}{\sin \rho}$. This can be seen for $x=-N$ from the formula $\varphi_{\alpha}(y)_{n+1}=\frac{\alpha^{2}-1}{\alpha^{2}+1}$ for $y \in \partial S_{N}^{n}$, which implies $\operatorname{dist}_{S^{n}}\left(\varphi_{\alpha}(y),-N\right)=\sin ^{-1}\left(\frac{2 \alpha}{\alpha^{2}+1}\right)$.

Proof of Theorem 3.3.. Since $\lim _{x \rightarrow \partial \Omega} u_{o}(x)=\infty$, given any $x \in \Omega$, there exists $\rho(x)>0$ such that

$$
\varphi_{\alpha(x)}^{-x}\left(\left\{y \in S^{n}:\langle y, x\rangle \geq 0\right\}\right)=B_{\rho(x)}(x) \subset \Omega
$$

and

$$
\left(\varphi_{\alpha(x)}^{-x}\right)^{*} g_{o} \geq \rho^{*}\left(\varphi_{\alpha(x)}^{-x}\right)^{*} g_{o}
$$

on $\left\{y \in S^{n}:\langle y, x\rangle \leq 0\right\}$, where $\alpha(x)=\frac{1-\cos \rho(x)}{\sin \rho(x)}$. Since $\lim _{x \rightarrow \partial \Omega} u(x, t)=\infty$ for all $t \in[0, T)$, the reflection method can be applied and we obtain

$$
\left(\varphi_{\alpha(x)}^{-x}\right)^{*} g(t) \geq \rho^{*}\left(\varphi_{\alpha(x)}^{-x}\right)^{*} g(t)
$$

on $\left\{y \in S^{n}:\langle y, x\rangle \leq 0\right\}$ for all $t \in[0, T)$. Hence

$$
\langle\nabla u(y, t), \nu\rangle \leq \frac{1-\alpha(x)^{2}}{\alpha(x)}=2 \cot \rho(x)
$$

for all $y \in \partial B_{\rho(x)}(x), t \in[0, T)$, and where $\nu$ is the unit inward normal to $\partial B_{\rho(x)}(x)$. Now let $K$ be a compact subset of $\Omega$. Then $\operatorname{dist}_{S^{n}}\left(K, S^{n} \sim\right.$ $\Omega):=\delta>0$ and we consider the $\delta / 2$-neighborhood of $K$ :

$$
N_{\delta / 2}(K)=\left\{z \in S^{n}: \operatorname{dist}_{S^{n}}(z, K) \leq \delta / 2\right\}
$$


which is compact in $\Omega$. Hence there exists a constant $\rho(K)>0$ such that for any $x \in N_{\delta / 2}(K)$

$$
\langle\nabla u(y, t), \nu\rangle \leq 2 \cot \rho(K)
$$

for all $y \in \partial B_{\rho(K)}(x), t \in[0, T)$. Since $\rho(K)<\delta / 2$, we conclude that

$$
|\nabla u(y, t)| \leq 2 \cot \rho(K)
$$

for all $y \in K$ and $t \in[0, T)$.

Corollary 3.4. Let $\left(M^{n}, \bar{g}\right)$ be a compact locally conformally flat manifold with positive scalar curvature. Let $g(x, t)=e^{u(x, t)} \bar{g}(x)$ be a solution to equation (3.3) on $M^{n} \times[0, T)$ with $g(0)=g_{o}=e^{u_{o}} \bar{g}$, an arbitrary initial metric. Then there exists a constant $C$ depending only on $g_{o}$ and $\bar{g}$ such that

$$
|\nabla u(x, t)| \leq C
$$

for all $(x, t) \in M^{n} \times[0, T)$.

Acknowledgement. We would like to thank Bob Gulliver for helpful discussions, especially for pointing out Corollary 2.4. Very special thanks to my father Yutze Chow for providing the wonderfully done PicTeX figures. I would also like to thank Lii-Perng Liou for discovering several misprints in an earlier version of this manuscript.

\section{References.}

[CG1] B. Chow and R. Gulliver, Aleksandrov reflection and nonlinear evolution equations, I: The n-sphere and n-ball, Cal. Var. 4 (1996), 249-264.

[CG2] B. Chow and R. Gulliver, Aleksandrov reflection and geometric evolution of embedded hypersurfaces, in preparation (1997).

[CT1] B. Chow and D.H. Tsai, Geometric expansion of convex plane curves, J. Diff. Geom. 44 (1996), 312-330.

[CT2] B. Chow and D.H. Tsai, Expansion of convex hypersurfaces by nonhomogeneous functions of curvature, preprint (1997).

[CLT] B. Chow, L.P. Liou and D.H. Tsai, On the nonlinear parabolic equation $u_{t}=F(\Delta u+n u)$ on $S^{n}$, Comm. Anal. Geom. 4 (1996), 415-434.

[G] C. Gerhardt, Flow of nonconvex hypersurfaces into spheres, J. Diff. Geom. 32 (1990), 299-314. 
Geometric Aspects of Aleksandrov Reflection and Gradient Estimates 409

[GNN] B. Gidas, W.-M. Ni and L. Nirenberg, Symmetry and related properties via the maximum principle, Comm. Math. Phys. 68 (1979), 209-243.

[H] G. Huisken, On the expansion of convex hypersurfaces by the inverse of symmetric functions, preprint (1988).

[L] L.P. Liou, Conformal flows by curvature on compact manifolds, submitted to Comm. Anal. Geom. 4(1996), 435-457.

[S1] R. Schoen, On the number of constant scalar curvature metrics in a conformal class, in Diff. Geom.: A symposium in honor of Manfredo do Carmo, H.B. Lawson and K. Tenenblat, eds., Pitman monographs, Wiley, (1991), 311-320.

[S2] R. Schoen, Course notes, Stanford University and NYU (1988-1990).

[SY] R. Schoen and S.T. Yau, Conformally flat manifolds, Kleinian groups and scalar curvature, Invent. Math. 92 (1988) , 47-71.

[T] D.H. Tsai, Geometric expansion of starshaped plane curves, 4 (1996), 459480.

[U1] J.I.E. Urbas, An expansion of convex hypersurfaces, J. Diff. Geom. 33 (1991), 91-125; Correction to, ibid. 35 (1992), 763-765.

[U2] J.I.E. Urbas, On the expansion of starshaped hypersurfaces by symmetric functions of their principal curvatures, Math. Z. 205 (1990), 355-372.

[Y] R. Ye, Global existence and convergence of the Yamabe flow, J. Diff.Geom. 39 (1994), 35-50.

RECEIVED OCTOBER 11, 1995.

UNIVERSITY OF MINNESOTA

SCHOOL OF MATHEMATICS

MINNEAPOLIS, MN 55455 